\title{
Effects of Wearing Facemasks During Brisk Walks: A COVID-19 Dilemma
}

\author{
Ophir Bar-On, MD, Yulia Gendler, PhD, Patrick Stafler, MD, Hagit Levine, MD, \\ Guy Steuer, MD, Einat Shmuel, MD, Dario Prais, MD, and Meir Mei-Zahav, MD
}

Background: During the Coronavirus disease 2019 (COVID-19) pandemic, wearing facemasks became obligatory worldwide.

Objectives: The objective of this study was to evaluate the effects of facemasks on gas exchange.

Methods: Healthy adults were assessed at rest and during slow and brisk 5-minute walks, with and without masks. We monitored $\mathrm{O}_{2}$ saturation, end-tidal carbon dioxide $\left(\mathrm{EtCO}_{2}\right)$, and heart and respiratory rates. Participants graded their subjective difficulty and completed individual sensations questionnaires.

Results: Twenty-one participants with a median age of 38 years (range, 29-57 years) were recruited. At rest, all vital signs remained normal, without and with masks. However, during slow and brisk walks, $\mathrm{EtCO}_{2}$ increased; the rise was significantly higher while wearing masks: slow walk, mean $\mathrm{EtCO}_{2}(\mathrm{mmHg})$ change $+4.5 \pm 2.4$ versus $+2.9 \pm 2.3, P=.004$; brisk walk $\mathrm{EtCO}_{2}$ change $+8.4 \pm 3.0$ versus $+6.2 \pm 4.0, P=.009$, with and without masks, respectively. Wearing masks was also associated with higher proportions of participant hypercarbia $\left(\mathrm{EtCO}_{2}\right.$ range, $\left.46-49 \mathrm{mmHg}\right)$ compared with walking without masks, though this was only partially significant. Mean $\mathbf{O}_{2}$-saturation remained stable (98\%) while walking without masks but decreased by $1.2 \% \pm 2.2$ while walking briskly with a mask $(P=.01)$. Mild desaturation $\left(\mathrm{O}_{2}\right.$ range, $93 \%$ to $\left.96 \%\right)$ was noted during brisk walks among $43 \%$ of participants with masks, compared with only $14 \%$ without masks $(P=.08)$. Borg's scale significantly increased while walking with a mask, for both slow and brisk walks $(P<.001)$. Sensations of difficulty breathing and shortness of breath were more common while walking with masks.

Conclusion: While important to prevent viral spread, wearing facemasks during brisk 5-minute walks might be associated with mild hypercarbia and desaturation. The clinical significance of these minor gas exchange abnormalities is unclear and should be further investigated. ( $\mathrm{J}$ Am Board Fam Med 2021;34:798-801.)

Keywords: COVID-19, Dyspnea, Exercise, Masks, Oxygen, Respiration Disorders, Surveys and Questionnaires

\section{Introduction}

During the Coronavirus disease 2019 (COVID-19) pandemic, facemasks became ubiquitous worldwide to protect against viral spread. ${ }^{1-3}$ Concurrently, warnings against mask potential harmful effects,

This article was externally peer reviewed.

Submitted 24 October 2020; revised 24 February 2021; accepted 11 March 2021.

From the Pulmonary Institute, Schneider Children's Medical Center of Israel, Petach Tikva, Israel (OB-O, YG, PS, HL, GS, ES, DP, MM-Z); Sackler Faculty of Medicine, Tel Aviv University, Tel Aviv, Israel (PS, HL, DP, MM-Z); The Department of Nursing, Ariel University, Ariel, Israel (YG).

Funding: None.

Conflict of interest: None.

Corresponding author: Meir Mei-Zahav, MD, Pulmonary Institute, Schneider Children's Medical Center of Israel, 14 Kaplan st. Petach Tikva, Israel 4920235, Phone: 972-39253654 (E-mail: mmeizahav@gmail.com). including hypoxemia and hypercarbia, appeared. The World Health Organization stated ${ }^{4}$ that "people should not wear masks while exercising," yet no real-life experimental supporting evidence was found. This study evaluated the effects of a standard surgical facemask on respiratory physiology in healthy individuals.

\section{Methods}

Healthy adult volunteers were recruited. Vital signs, oxygenation, and ventilation were monitored under 3 different conditions: (a) at rest, (b) during a slow walk $(4 \mathrm{~km} / \mathrm{h})$, and (c) during a brisk walk $(7 \mathrm{~km} / \mathrm{h})$, with the latter 2 on a standard treadmill at $0^{\circ}$ inclination. Each segment lasted 5 minutes, and each was performed twice—in random order-once 
without, and once with, a standard disposable surgical facemask (Non-Woven 3-ply FaceMask, LeJian Protective Equipment, China). A 5-minute recovery period was allocated between segments. The room settings were $25^{\circ} \mathrm{C}$ with $48 \%$ humidity.

Using a Welch Allyn 300 Vital Signs Monitor, we documented heart rate and oxygen saturation continuously; carbon dioxide was measured endtidally $\left(\mathrm{EtCO}_{2}\right)$ using LifeSense II (Nonin Medical Inc., Minneapolis, USA) via nasal prongs. This device also measured respiratory rate. Values were registered at 0 minutes, 2 minutes, 4 minutes, 5 minutes, and at recovery end.

Normal $\mathrm{CO}_{2}$ was defined as $35-45 \mathrm{mmHg}$; Normal oxygen saturation was defined as $\geq 97 \%$. $^{5,6}$

Participants rated their subjective difficulty using Borg's Exertional Scale, ${ }^{7}$ an established numeric tool (range, 1 to 10), measuring the combined feeling of effort and exertion, breathlessness, and fatigue during physical work. Furthermore, participants graded their individual sensations, comparing the same activity, with versus without a mask, namely: difficulty breathing, shortness of breath, "choking" feeling, headache, dizziness, and weakness.

Data were analyzed using SPSS, version 25 (SPSS Inc; Armonk, NY). Results are presented as mean \pm standard deviation (SD) or median + range according to variable distribution. For comparison of parameters measured with versus without a mask, paired-samples $t$-test was used when normal distribution was assumed; otherwise, a Wilcoxon ranksum test was used. A general linear model was used for repeated measurements analysis. Sample size was calculated using power analysis $(\alpha=0.05,1-\beta=0.8)$. The study was approved by the organization's Institutional Research Board (RMC0325-20), and participants signed informed consent before entering the study.

\section{Results}

Twenty-one volunteers were recruited into the study. Of the 21 volunteers, 11 were female, with a median age of 38 years (range, 29-57 years), median body mass index of 24.5 (range, 20-33.6), and without underlying cardiopulmonary morbidity. During the rest, all vital signs were within normal limits among the participants, with and without a mask.

During the slow walk, respiratory and heart rates increased equally with and without a mask among the participants. A small yet statistically significant difference in $\mathrm{EtCO}_{2}$ increase was observed while wearing a mask $(P=.004)$. Two examinees showed mild hypercarbia wearing a mask, while none without $(P<.001)$ as shown in Table 1.

During the brisk 5-minute walk, we saw a statistically significant increase in $\mathrm{EtCO}_{2}$, more profoundly while wearing a mask: a mean change of $8.4 \pm$ $3.0 \mathrm{mmHg}$ versus $6.2 \pm 4.0 \mathrm{mmHg}(P=.009)$, from baseline of $33.5 \pm 3.8$ without mask, $33.9 \pm 4.3$ with mask, respectively (Figure 1). Three participants (14\%) showed hypercarbia $\left(\mathrm{EtCO}_{2}>45 \mathrm{mmHg}\right.$; max, 48.5) while walking with a mask, compared with only $1(5 \%)\left(\mathrm{EtCO}_{2}=46 \mathrm{mmHg}\right)$ while walking without a mask $(P=.60)$. Mean oxygen saturation remained stable while walking without a mask (98\%) but decreased slightly, yet statistically significant, by $1.2 \% \pm 2.2$ while walking with a mask, from $97.9 \% \pm 2.3$ to $96.7 \% \pm 1.9(P=.01)$ (Figure 1$)$. Nine participants $(43 \%)$ demonstrated mild desaturation $\left(\mathrm{O}_{2}\right.$ range, $93 \%$ to $\left.96 \%\right)$ while walking with a mask, compared with only 3 (14\%) who had mild desaturation $\left(\mathrm{O}_{2}\right.$ range, $93 \%$ to $\left.96 \%\right)$ while walking without a mask $(P=.08)$. As anticipated for physical activity, heart and respiratory rates increased with and without a mask, similarly.

Table 1. Changes in Parameter Values, Comparing Slow and Brisk Walks, With a Mask Versus Without a Mask

\begin{tabular}{|c|c|c|c|c|c|c|}
\hline \multirow[b]{2}{*}{ Parameter (Mean \pm SD) } & \multicolumn{3}{|c|}{ Slow Walk } & \multicolumn{3}{|c|}{ Brisk Walk } \\
\hline & Without Mask & With Mask & $P$ value & Without Mask & With Mask & $P$ value \\
\hline $\mathrm{EtCO}_{2}(\mathrm{mmHg})$ change & $+2.9 \pm 2.3$ & $+4.5 \pm 2.4$ & .004 & $+6.2 \pm 4.0$ & $+8.4 \pm 3.0$ & .009 \\
\hline $\mathrm{O}_{2}$ saturation $\%$ change & $-0.2 \pm 1.2$ & $-0.2 \pm 0.9$ & $>.99$ & $+0.24 \pm 1.3$ & $-1.2 \pm 2.2$ & 01 \\
\hline $\mathrm{EtCO}_{2}>45 \mathrm{mmHg}, \mathrm{n}(\%)$ & $0(0.0)$ & $2(9.5)$ & $<.001$ & $1(5.0)$ & $3(14.0)$ & 60 \\
\hline $\mathrm{O}_{2}$ saturation $<97 \%, \mathrm{n}(\%)$ & $6(28.0)$ & $3(14.0)$ & .45 & $3(14.0)$ & $9(43.0)$ & .08 \\
\hline Borg Exertion Scale & $1.3 \pm 0.59$ & $1.8 \pm 0.94$ & .002 & $3.1 \pm 1.43$ & $4.3 \pm 1.6$ & $<.001$ \\
\hline
\end{tabular}

$\mathrm{EtCO}_{2}$, end-tidal carbon dioxide; SD, standard deviation.

${ }^{*} P$ values calculated for trend of repeated measurements using a general linear model. 
Figure 1. Mean $\mathrm{O}_{2}$-saturation (\%) and $\mathrm{EtCO}_{2}$, during a brisk 5-minute walk, with a mask versus without a mask. Abbreviation: EtCO2, end-tidal carbon dioxide.

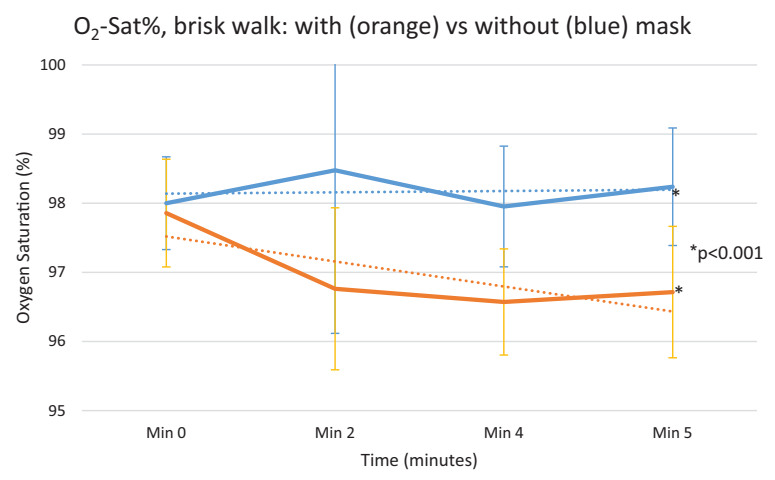

$\mathrm{EtCO}_{2}$, brisk walk: with (orange) vs without (blue) mask

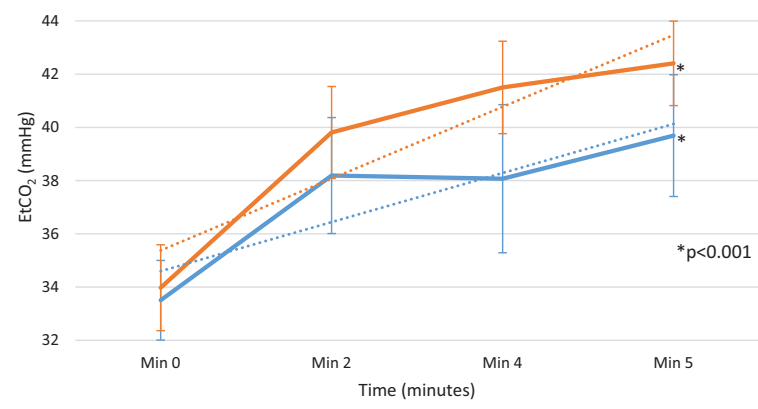

There was a statistically significant increase in the Borg Exertion Scale while walking with a mask for both slow and brisk walks (Table 1).

In the subjective perception questionnaire, participants described that walking briskly with a mask, compared with walking without a mask, caused "difficulty breathing" (86\%), "shortness of breath" (33\%), "choking" feeling (57\%), and "dizziness" (19\%); none of these were reported while walking without a mask.

\section{Discussion}

In this controlled trial, we demonstrated that wearing a facemask during a 5-minute walk has mild effects on respiratory parameters, causing $\mathrm{EtCO}_{2}$ to increase more profoundly than walking without a mask. In addition, oxygen saturation decreases while walking briskly with a facemask.

Most values remained within normal limits, but a subset of participants reached abnormal levels. The consequences and clinical significance of these minor gas exchange abnormalities are unclear, yet several participants reported shortness of breath and dizziness while walking briskly with a mask. The relationship between these phenomena has to be further investigated. Notably, our evaluation tested healthy volunteers and lasted only 5 minutes in an air-conditioned room.

$\mathrm{EtCO}_{2}$ probably increases due to rebreathing of expired air "trapped" in the chamber formed between the mouth and the mask. During exercise and the inevitable increase in respiratory rate coupled with increased $\mathrm{CO}_{2}$ production, there was a clearing of trapped air that was probably less effective, and perhaps even further dampened by mask warmth and humidity. This activity also increases resistance and overall work of breathing, contributing to subjective sensations of breathlessness, and increased Borg scale. A recent article by Fikenzer et $\mathrm{al}^{8}$ also demonstrated a marked negative impact of masks on exercise capacity.

These effects of breathing through a mask have not been thoroughly investigated. Kim et $\mathrm{al}^{9}$ evaluated volunteers walking calmly for 1 hour wearing an N95 mask. They observed an increase in transcutaneous $\mathrm{CO}_{2}$ (with most results remaining within normal limits), but no change in oxygen saturation. Person et $\mathrm{al}^{10}$ examined the effect of wearing a facemask on healthy individuals while performing a calm 6-Minute-Walk-Test. Distance walked, oxygen saturation, heart rate, and respiratory rate did not differ while wearing a mask. Samannan et $\mathrm{al}^{11}$ recently reported a minor influence of masks on gas exchange in healthy volunteers, and COPD patients, performing 6-minute walk tests. Rebmann et $\mathrm{al}^{12}$ evaluated nurses wearing facemasks and did not see any clinically relevant physiologic changes, although many subjective symptoms were reported.

\section{Summary}

Wearing masks is safe and important to prevent viral spread. That said, we demonstrated that wearing a surgical mask causes a mild, yet statistically significant, increase in $\mathrm{EtCO}_{2}$, as well as mild $\mathrm{O}_{2}$ desaturation, in healthy adults performing a brisk 5minute walk in a comfortable setting.

While the abnormalities in our study may not be clinically significant, these statistically significant changes should encourage further evaluation of whether masks may cause additional gas exchange abnormalities during longer activity, especially in older people and those with comorbidities. 
We thank the Gerner family who donated the Nonin LifeSense II $\mathrm{EtCO}_{2}$ monitor to the Pulmonary Institute at Schneider Children's Medical Center of Israel, in memory of their son $\mathrm{Oz}$, who died of cystic fibrosis.

To see this article online, please go to: http://jabfm.org/content/ 34/4/798.full.

\section{References}

1. Liang M, Gao L, Cheng C, et al. Efficacy of face mask in preventing respiratory virus transmission: A systematic review and meta-analysis. Travel Med Infect Dis 2020;36:101751. doi:10.1016/j.tmaid.2020.101751.

2. Greenhalgh T, Schmid MB, Czypionka T, Bassler D, Gruer L. Face masks for the public during the covid-19 crisis. BMJ 2020;369:1-4. doi:10.1136/ bmj.m1435.

3. Brooks JT, Butler JC, Redfield RR. Universal masking to prevent SARS-CoV-2 transmissionthe time is now. JAMA 2020;324:635.

4. World Health Organization (WHO). WHO Myth Busters. Coronavirus disease (COVID-19) advice for the public: Mythbusters. Published 2020. Available from:https://www.who.int/emergencies/diseases/novelcoronavirus-2019/advice-for-public/myth-busters\# exercising. Accessed February 23, 2021.
5. Guyton A, Hall J. In: Textbook of Medical Physiology. 12th ed. Elsevier; 2010:495-502.

6. Levitzky M. In: Pulmonary Physiology. 7th ed. The McGraw-Hill Companies, Inc; 2007.

7. Borg G. Ratings of perceived exertion and heart rates during short-term cycle exercise and their use in a new cycling strength test. Int J Sports Med 1982;3:153-158.

8. Fikenzer S, Uhe T, Lavall D, et al. Effects of surgical and FFP2/N95 face masks on cardiopulmonary exercise capacity. Clin Res Cardiol 2020;109:1522-1530.

9. Kim JH, Benson SM, Roberge RJ. Pulmonary and heart rate responses to wearing N95 filtering facepiece respirators. Am J Infect Control 2013;41:24-27.

10. Person E, Lemercier C, Royer A, Reychler G. Effect of a surgical mask on six minute walking distance. Rev Mal Respir 2018;35:264-268.

11. Samannan R, Holt G, Calderon-Candelario R, Mirsaeidi M, Campos M. Effect of face masks on gas exchange in healthy persons and patients with COPD. Ann Am Thorac Soc 2020;18:1-7.

12. Rebmann T, Carrico R, Wang J. Physiologic and other effects and compliance with long-term respirator use among medical intensive care unit nurses. Am J Infect Control 2013;41:1218-1223. 